Preprints of the

Max Planck Institute for

Research on Collective Goods

Bonn 2011/7

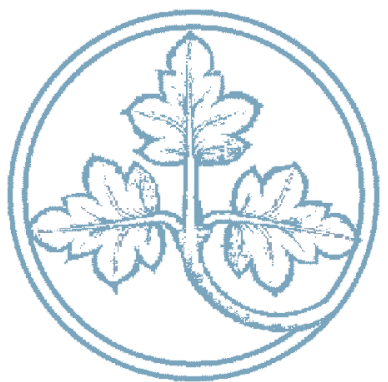

From Posteriors to Priors via Cycles: An Addendum

Martin Hellwig

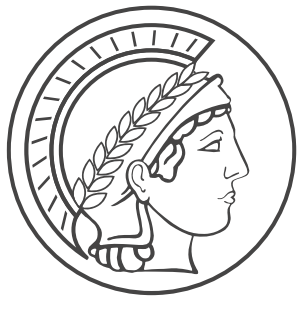




\title{
From Posteriors to Priors via Cycles: An Addendum
}

\author{
Martin Hellwig
}

May 2011 


\title{
From Posteriors to Priors via Cycles: An Addendum*
}

\author{
Martin F. Hellwig \\ Max Planck Institute for Research on Collective Goods \\ Kurt-Schumacher-Str. 10, D- 53113 Bonn, Germany \\ hellwig@coll.mpg.de
}

April 21, 2011

\begin{abstract}
Rodrigues-Neto (2009) has shown that a given specification of posteriors of different players in an incomplete-information setting is compatible with a common prior if and only if the posteriors satisfy the so-called cycle equations. This note shows that, if, for any player, any element of the partition of the this player has a nonempty intersection with any element of the partition of any other player, then it suffices to verify the cycle equations for all cycles of length 4 or less.

Key Words: Belief systems, consistency, common priors, cycle equations.

JEL: D82, D83.
\end{abstract}

\section{The Basic Idea}

In a recent note, Rodrigues-Neto [4] has developed an algebraic approach to verifying whether a given specification of posteriors for different players is compatible with a common prior. ${ }^{1}$ The algebraic approach provides an alternative to the syntactic approach of [1], as well as the semantic approaches that are based on separation theorems ([3], [6]) or on limits of iterated expectations in Markov chains ([5]). In a model with finitely many players and a finite state space, the algebraic approach requires the verification that the posteriors satisfy a given set of equations, the so-called cycle equations. This note shows that, if, for any two different players, any element of the partitions of the first player has a nonempty intersection with any element of the partitiion of the second player, then it is actually enough to verify these equations for cycles of length 4 or less.

\footnotetext{
*For helpful discussions and comments, I thank Sophie Bade, Christoph Engel, Yossi Feinberg, Alia Gizatulina, and Christian Hellwig.

${ }^{1}$ For a similar result, see Proposition 4 in Hellman and Samet [2].
} 
The procedure for verifying that posteriors are compatible with a common prior is thus further simplified.

To understand the contribution, consider Figure 1, a version of RodriguesNeto's meet-join diagram. There are two players and nine states of nature. The different states are entered in a square matrix so as to reflect the players' information partititions. Player 1's partition corresponds to the rows of the matrix, player 2's partition to the columns. The column to the left of the matrix indicates the elements of player 1's partition, the row at the top the elements of player 2's partition.

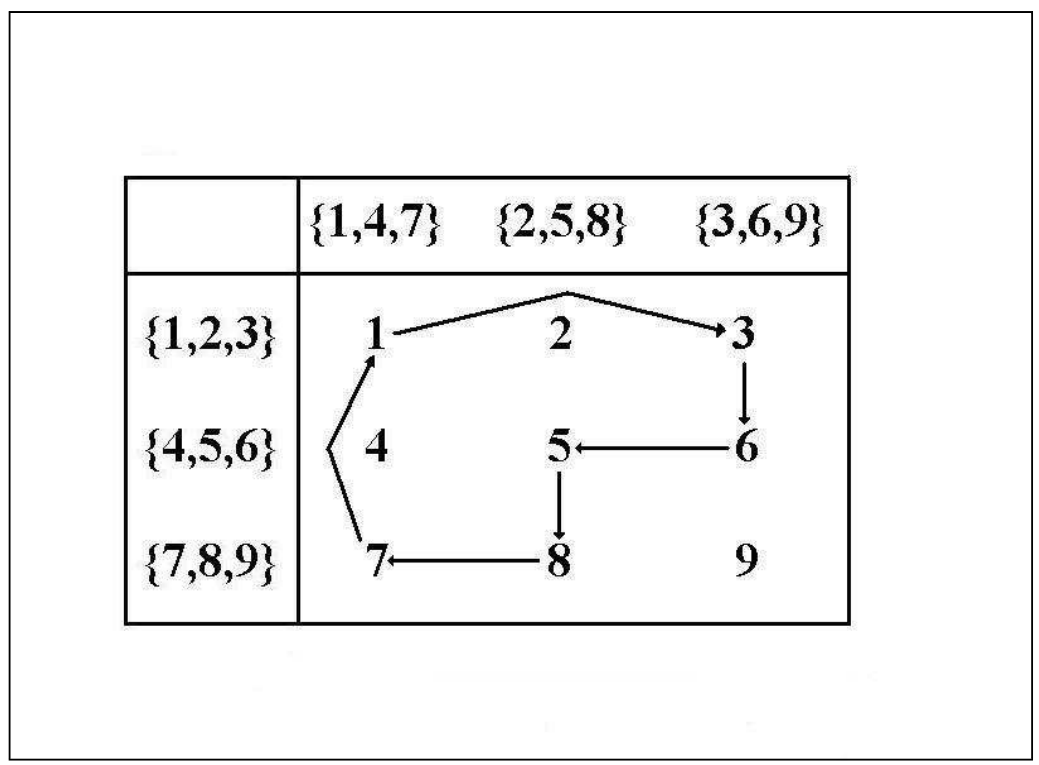

Figure 1

In this figure, an oriented horizontal edge leads from one state to another state in the same row; an oriented vertical edge leads from one state to another state in the same column. A cycle is given by a sequence of connected horizontal and vertical edges that leads back to the initial state. An example is given by the sequence of arrows in the figure that lead from state 1 to state 3 , from state 3 to state 6 , from state 6 to state 5 , from state 5 to state 8 , from state 8 to state 7 , and from state 7 back to state 1 .

The different oriented edges are given different weights. The weight of an edge leading from a state $\omega_{1}$ to a state $\omega_{2}$ is defined as the posterior probability of the state $\omega_{2}$, as seen by the player who is unable to distinguish between $\omega_{1}$ and $\omega_{2}$. Thus, in Figure 1, the weight of edge $1 \rightarrow 3$ is equal to the posterior probability of state 3 , as seen from the perspective of player 1 when he knows that the state belongs to the set $\{1,2,3\}$. The weight of the reverse edge $3 \rightarrow 1$ is equal to the posterior probability of state 1 from the perspective of player 1 when the state belongs to $\{1,2,3\}$. 
A cycle equation requires that the product of the weights of edges along a given cycle be the same as the product of the weights of edges along the reverse cycle. Thus, in Figure 1, the product of weights of edges in the cycle $1 \rightarrow 3 \rightarrow 6 \rightarrow 5 \rightarrow 8 \rightarrow 7 \rightarrow 1$ must be the same as the product of weights of edges in the reverse cycle $1 \rightarrow 7 \rightarrow 8 \rightarrow 5 \rightarrow 6 \rightarrow 3 \rightarrow 1$. This requirement is nontrivial because the weight assigned to any one edge is usually not the same as the weight assigned to the reverse edge. For example, there is no reason why, in Figure 1, the weights of the edges $1 \rightarrow 3$ and $3 \rightarrow 1$, i.e. the posterior probabilities of states 3 and 1 , as seen from the perspective of player 1 when the state belongs to $\{1,2,3\}$, should be the same.

Rodrigues-Neto [4] shows that a given system of posteriors for different agents is compatible with a common prior if and only if the cycle equation is satisfied for every cycle. This note shows that, for an interesting class of case, the condition of Rodrigues-Neto is satisfied if and only if the cycle equation is satisfied for every cycle of length 4 or less.

The reason is that the different cycle equations are not independent. For any longer cycle, the validity of the cycle equation is implied by the validity of the cycle equations for shorter cycles. Thus, in the two-player example of Figure 1 , validity of the cycle equation for the cycle $1 \rightarrow 3 \rightarrow 6 \rightarrow 5 \rightarrow 8 \rightarrow 7 \rightarrow 1$, which has six edges, is implied by the cycle equations for the three cycles shown in Figure 2, namely, $1 \rightarrow 3 \rightarrow 6 \rightarrow 4 \rightarrow 1,1 \rightarrow 4 \rightarrow 5 \rightarrow 2 \rightarrow 1$, and $1 \rightarrow 2 \rightarrow 8 \rightarrow 7 \rightarrow 1$, all of which have four edges.

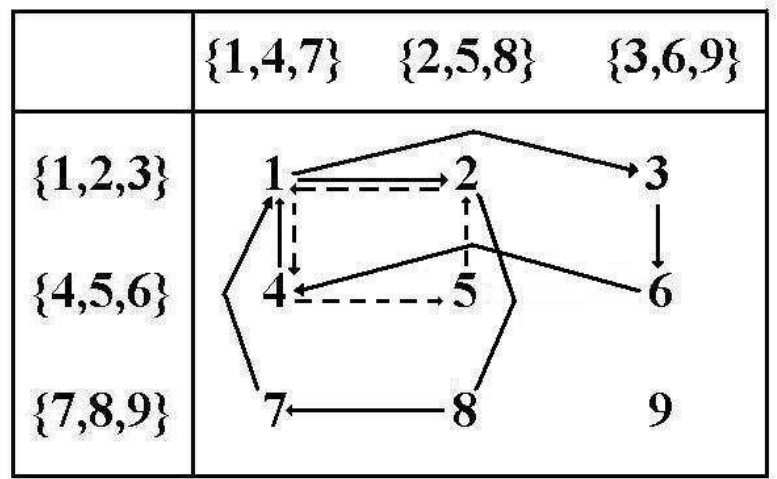

Figure 2

We can think of the cycle $1 \rightarrow 3 \rightarrow 6 \rightarrow 5 \rightarrow 8 \rightarrow 7 \rightarrow 1$ in Figure 1 as being "mimicked" by the cycle $1 \rightarrow 3 \rightarrow 6 \rightarrow 4 \rightarrow 1 \rightarrow 4 \rightarrow 5 \rightarrow 2 \rightarrow$ 
$1 \rightarrow 2 \rightarrow 8 \rightarrow 7 \rightarrow 1$, which is obtained by joining the three shorter cycles $1 \rightarrow 3 \rightarrow 6 \rightarrow 4 \rightarrow 1,1 \rightarrow 4 \rightarrow 5 \rightarrow 2 \rightarrow 1$, and $1 \rightarrow 2 \rightarrow 8 \rightarrow 7 \rightarrow 1$ in Figure 2. The word "mimicked" here must be taken with a grain of salt. Some of the edges of the cycle in Figure 1, namely $6 \rightarrow 5$ and $5 \rightarrow 8$, are not also edges of the cycles in Figure 2. Moreover, some of the edges of the cycles in Figure 2 are not also edges of the cycle in Figure 1. However, the "missing" edges $6 \rightarrow 5$ and $5 \rightarrow 8$ in the cycle $1 \rightarrow 3 \rightarrow 6 \rightarrow 5 \rightarrow 8 \rightarrow 7 \rightarrow 1$ in Figure 1 have counterparts in the shorter cycles that have exactly the same weights and therefore make exactly the same contributions to the cycle equation. Specifically, the weights of the edges $6 \rightarrow 5$ in Figure 1 and $4 \rightarrow 5$ in Figure 2 are the same; they are both given by the conditional probability that player 1 assigns to the state 5 given the information that the state belongs to the set $\{4,5,6\}$. Similarly, the weights of the edge $5 \rightarrow 8$ in Figure 1 and $2 \rightarrow 8$ in Figure 2 are also the same. Once these equivalences are taken into account, the "excess" of edges in Figure 2 over the cycle $1 \rightarrow 3 \rightarrow 6 \rightarrow 5 \rightarrow 8 \rightarrow 7 \rightarrow 1$ in Figure 1 is seen to be redundant in the sense that, e.g., the edges $4 \rightarrow 1$ and $1 \rightarrow 4$ just neutralize each other because their combined contributions to the two sides of the cycle equation is exactly the same. Therefore, the cycle equation for the cycle $1 \rightarrow 3 \rightarrow 6 \rightarrow 5 \rightarrow 8 \rightarrow 7 \rightarrow 1$ in Figure 1 is equivalent to the cycle equation for the more roundabout cycle $1 \rightarrow 3 \rightarrow 6 \rightarrow 4 \rightarrow 1 \rightarrow 4 \rightarrow 5 \rightarrow 2 \rightarrow 1 \rightarrow 2 \rightarrow 8 \rightarrow 7 \rightarrow 1$ in Figure 2 , which in turn is implied by the cycle equations for the shorter cycles $1 \rightarrow 3 \rightarrow 6 \rightarrow 4 \rightarrow 1$, $1 \rightarrow 4 \rightarrow 5 \rightarrow 2 \rightarrow 1$, and $1 \rightarrow 2 \rightarrow 8 \rightarrow 7 \rightarrow 1$.

This finding reflects a general principle, which is applicable whenever a cycle with more than four edges can be mimicked in this way by a combination of shorter cycles.

A mimicking of longer cycles by combinations of shorter cycles is not always possible. An example is provided by the six-edge cycle $1 \rightarrow 2 \rightarrow 3 \rightarrow 4 \rightarrow 5 \rightarrow$ $6 \rightarrow 1$ in Figure 3. Whereas in Figures 1 and 2, each element of the partition of player 2 can be reached from each element of the partition of player 1 , and vice versa, in Figure 3, for example, the element $\{2,3\}$ of the partition of player 2 cannot be reached from the element $\{5,6\}$ of player $1 .^{2}$

\footnotetext{
${ }^{2}$ At first sight, the notion that certain elements of the partition of player 2 cannot be reached from a given element of the partition of player 1 seems to depend on the particular representation of states, events, and information in Figure 3. For suppose that we add three states, (Row 1, Column 3), (Row 2, Column 1), (Row 3, Column 2), to the six-state model of Figure 2, while maintaining the interpretation of rows and columns as representing the players' respective partitions. Then the element Column 2 of player 2's partition can be reached from the element Row 3 of player 1's partition. However, in this extended model, conditional probabilities of the state (Row 3, Column 2) from the perspectives of both players must be equal to zero. If conditional probabilities can be equal to zero, the mimicking operation in Figure 1 does not work. For example, if, in Figure 1, both player 1 and player 2 assign conditional probability zero to state 4 , then the cycle equation for the roundabout cycle $1 \rightarrow 3 \rightarrow 6 \rightarrow 4 \rightarrow 1 \rightarrow 4 \rightarrow 5 \rightarrow 2 \rightarrow 1 \rightarrow 2 \rightarrow 8 \rightarrow 7 \rightarrow 1$ is trivially satisfied but this does not permit any inference about the cycle equation for $1 \rightarrow 3 \rightarrow 6 \rightarrow 5 \rightarrow 8 \rightarrow 7 \rightarrow 1$. Rodrigues-Neto [4] assumes that all relevant conditional probabilities are strictly positive.
} 


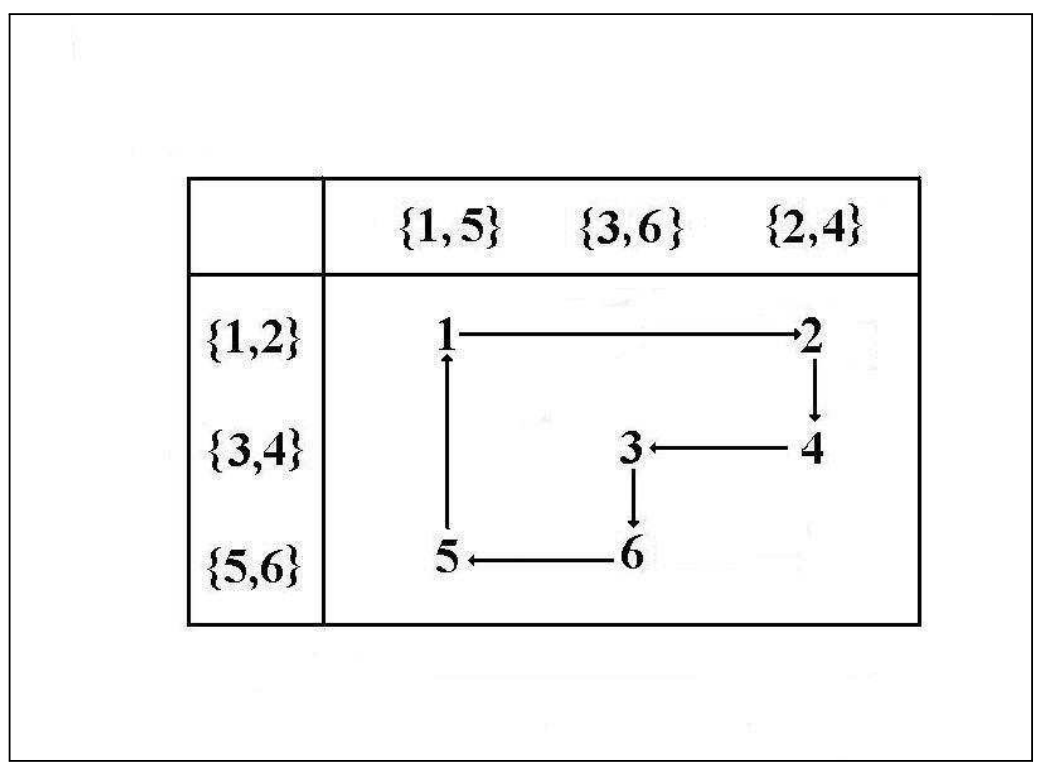

Figure 3

If each element of a player's partition can be reached from each element of any other player's partition, such a constellation cannot arise. In the following, I will show that any specification of posteriors which satisfies this condition is compatible with a common prior if and only if the cycle equation is satisfied for every cycle of length 4 or less.

\section{Formal Statement and Proof of the Result}

The framework of the formal analysis is the same as in Rodrigues-Neto [4]. There are $J$ players $j=1, \ldots, J$. There are also $n$ possible states of nature $\omega=1, \ldots, n$. Each player $j$ 's information is characterized by a partition $\Pi^{j}=\left\{\pi_{1}^{j}, \ldots, \pi_{\ell^{j}}^{j}\right\}$ of the state space $S=\{1,2, \ldots, n\}$. A posterior for player $j$ is a vector $\theta^{j}=$ $\left(\theta_{1}^{j}, \ldots \theta_{n}^{j}\right)$, where, for each state $\omega, \theta_{\omega}^{j}>0$ is the posterior probability that player $j$ assigns to the state $\omega$ when he is informed that the true state belongs to the unique element $\pi^{j}(\omega)$ of the partition $\Pi^{j}$ that contains $\omega$. For any set $\pi_{k}^{j} \in \Pi^{j}$, obviously, $\sum_{\omega \in \pi_{k}^{j}} \theta_{\omega}^{j}=1$.

The posteriors $\theta^{1}, \theta^{2}, \ldots, \theta^{J}$ are compatible with a common prior $\mu$ if and only if, for all $j$ and all $\omega$,

$$
\theta_{\omega}^{j}=\frac{\mu(\omega)}{\sum_{\omega^{\prime} \in \pi^{j}(\omega)} \mu\left(\omega^{\prime}\right)} .
$$

The question is under what conditions on $\theta^{1}, \theta^{2}, \ldots, \theta^{J}$ such a prior exists.

To answer this question, Rodrigues-Neto introduces the concepts of (oriented) edge and cycle. An edge is an ordered triple $\left(j, \omega_{p}, \omega_{q}\right)$ such that $\omega_{p}$ 
and $\omega_{q}$ are two states belonging to the same element of player $j$ 's partition, i.e. $\pi^{j}\left(\omega_{p}\right)=\pi^{j}\left(\omega_{q}\right)$. A cycle is a finite sequence of edges, $\left\{\left(j^{k}, \omega_{p}^{k}, \omega_{q}^{k}\right)\right\}_{k=1}^{K}$ such that, for $k=1, \ldots K-1, \omega_{q}^{k}=\omega_{p}^{k+1}$ and, moreover, $\omega_{q}^{K}=\omega_{p}^{1}$. The opposite of the edge $\left(j, \omega_{p}, \omega_{q}\right)$ is the edge $\left(j, \omega_{q}, \omega_{p}\right)$ that involves the same player but has the order of states reversed. The opposite of the cycle $\left\{\left(j^{1}, \omega_{p}^{1}, \omega_{q}^{1}\right), \ldots,\left(j^{K}, \omega_{p}^{K}, \omega_{q}^{K}\right)\right\}$ is the cycle $\left\{\left(j^{K}, \omega_{q}^{K}, \omega_{p}^{K}\right), \ldots,\left(j^{1}, \omega_{q}^{1}, \omega_{p}^{1}\right)\right\}$ that is obtained by taking the opposites of the edges $\left(j^{1}, \omega_{p}^{1}, \omega_{q}^{1}\right), \ldots,\left(j^{K}, \omega_{p}^{K}, \omega_{q}^{K}\right)$ and reversing their order.

The oriented edge $\left(j, \omega_{p}, \omega_{q}\right)$ is given the weight $\theta_{\omega_{q}}^{j}$. Note that this weight is independent of $\omega_{p}$. Independence of $\omega_{p}$ reflects the fact that the conditional probability which player $j$ assigns to the state $\omega_{q}$ is the same for all states $\omega_{p}$ that belong to the same element $\pi^{j}\left(\omega_{q}\right)$ as the state $\omega_{q}$ itself.

Given the weights $\theta_{\omega_{q}}^{j}$ for different edges, the product of weights of edges in the cycle $\left\{\left(j^{k}, \omega_{p}^{k}, \omega_{q}^{k}\right)\right\}_{k=1}^{K}$ is $\prod_{k=1}^{K} \theta_{\omega_{q}^{k}}^{j^{k}}$, the product of weights for the opposite cycle, $\prod_{k=1}^{K} \theta_{\omega_{p}^{k}}^{j^{k}}$. The following result is proved in Rodrigues-Neto [4].

Proposition 1 (Rodrigues-Neto) The posteriors $\theta^{1}, \theta^{2}, \ldots, \theta^{J}$ are compatible with some common prior $\mu$ if and only if the cycle equation

$$
\prod_{k=1}^{K} \theta_{\omega_{q}^{k}}^{j^{k}}=\prod_{k=1}^{K} \theta_{\omega_{p}^{k}}^{j^{k}}
$$

holds for every cycle $\left\{\left(j^{k}, \omega_{p}^{k}, \omega_{q}^{k}\right)\right\}_{k=1}^{K}$.

Building on this result, I obtain:

Proposition 2 Assume that, for all players $j, k \neq j$, all $\pi^{j} \in \Pi^{j}$ and all $\pi^{k} \in \Pi^{k}, \pi^{j} \cap \pi^{k} \neq \emptyset$. Then the posteriors $\theta^{1}, \theta^{2}, \ldots, \theta^{J}$ are compatible with some common prior $\mu$ if and only if the cycle equation (2) holds for every cycle $\left\{\left(j^{k}, \omega_{p}^{k}, \omega_{q}^{k}\right)\right\}_{k=1}^{K}$ with $K \leq 4$.

Proof. Trivially, the result of Rodrigues-Neto implies that, the posteriors $\theta^{1}, \theta^{2}, \ldots, \theta^{J}$ are compatible with a common prior $\mu$, then the cycle equation (2) must hold for every cycle $\left\{\left(j^{k}, \omega_{p}^{k}, \omega_{q}^{k}\right)\right\}_{k=1}^{K}$ with $K \leq 4$. To prove that, under the additional assumption on the partitions $\Pi^{1}, \ldots, \Pi^{J}$, the converse is also true, I will show that, if the cycle equation (2) holds for every cycle $\left\{\left(j^{k}, \omega_{p}^{k}, \omega_{q}^{k}\right)\right\}_{k=1}^{K}$ with $K \leq 4$, then it also holds for every cycle $\left\{\left(j^{k}, \omega_{p}^{k}, \omega_{q}^{k}\right)\right\}_{k=1}^{K}$ with $K>4$.

The argument proceeds by induction on $K$. Suppose that the cycle equation holds for every cycle $\left\{\left(j^{k}, \omega_{p}^{k}, \omega_{q}^{k}\right)\right\}_{k=1}^{K^{\prime}}$ with $K^{\prime} \leq K-1$, and consider any cycle $\left\{\left(j^{k}, \omega_{p}^{k}, \omega_{q}^{k}\right)\right\}_{k=1}^{K}$ with $K$ edges. Fix some $\hat{\jmath} \neq j^{3}$. Using the assumption that $\pi^{j} \cap \pi^{k} \neq \emptyset$ for all $\pi^{j} \in \Pi^{j}$ and all $\pi^{k} \in \Pi^{k}$, for all $j$ and $k \neq j$, let $\hat{\omega}$ be an element of $\pi^{j^{3}}\left(\omega_{p}^{3}\right) \cap \pi^{\hat{\jmath}}\left(\omega_{q}^{K}\right)$. Since $\pi^{j^{3}}\left(\omega_{p}^{3}\right)=\pi^{j^{3}}\left(\omega_{q}^{3}\right), \hat{\omega}$ is also an element of $\pi^{j^{3}}\left(\omega_{q}^{3}\right) \cap \pi^{\hat{\jmath}}\left(\omega_{q}^{K}\right)$. Thus, $\left(j^{3}, \omega_{p}^{3}, \hat{\omega}\right),\left(\hat{\jmath}, \hat{\omega}, \omega_{q}^{K}\right),\left(\hat{\jmath}, \omega_{q}^{K}, \hat{\omega}\right),\left(j^{3}, \hat{\omega}, \omega_{q}^{3}\right)$ 
are all admissible edges. If, in the cycle $\left\{\left(j^{k}, \omega_{p}^{k}, \omega_{q}^{k}\right)\right\}_{k=1}^{K}$, the edge $\left(j^{3}, \omega_{p}^{3}, \omega_{q}^{3}\right)$ is replaced by the four edges $\left(j^{3}, \omega_{p}^{3}, \hat{\omega}\right),\left(\hat{\jmath}, \hat{\omega}, \omega_{q}^{K}\right),\left(\hat{\jmath}, \omega_{q}^{K}, \hat{\omega}\right)$, and $\left(j^{3}, \hat{\omega}, \omega_{q}^{3}\right)$, the result is a $K+3$-edge cycle. ${ }^{3}$ This $K+3$-edge cycle is made up of two shorter cycles, the four-edge cycle $\left(j^{1}, \omega_{p}^{1}, \omega_{q}^{1}\right),\left(j^{2}, \omega_{p}^{2}, \omega_{q}^{2}\right),\left(j^{3}, \omega_{p}^{3}, \hat{\omega}\right),\left(\hat{\jmath}, \hat{\omega}, \omega_{q}^{K}\right)$, and the $K$-1-edge cycle $\left(\hat{\jmath}, \omega_{q}^{K}, \hat{\omega}\right),\left(j^{3}, \hat{\omega}, \omega_{q}^{3}\right),\left(j^{4}, \omega_{p}^{4}, \omega_{q}^{4}\right), \ldots,\left(j^{K}, \omega_{p}^{K}, \omega_{q}^{K}\right)$. By the induction hypothesis, each of these shorter cycles satisfies the cycle equation. Thus,

$$
\theta_{\omega_{q}^{1}}^{j^{1}} \cdot \theta_{\omega_{q}^{2}}^{j^{2}} \cdot \theta_{\hat{\omega}}^{j^{3}} \cdot \theta_{\omega_{q}^{K}}^{\hat{\jmath}}=\theta_{\hat{\omega}}^{\hat{\jmath}} \cdot \theta_{\omega_{p}^{3}}^{j^{3}} \cdot \theta_{\omega_{p}^{2}}^{j^{2}} \cdot \theta_{\omega_{p}^{1}}^{j^{1}}
$$

and

$$
\theta_{\hat{\omega}}^{\hat{\jmath}} \cdot \prod_{k=3}^{K} \theta_{\omega_{q}^{k}}^{j^{k}}=\prod_{k=4}^{K} \theta_{\omega_{p}^{k}}^{j^{k}} \cdot \theta_{\hat{\omega}}^{j^{3}} \cdot \theta_{\omega_{q}^{K}}^{\hat{\jmath}}
$$

Upon multiplying these equation by each other and rearranging terms, one obtains

$$
\theta_{\hat{\omega}}^{j^{3}} \cdot \theta_{\omega_{q}^{K}}^{\hat{\jmath}} \cdot \theta_{\hat{\omega}}^{\hat{\jmath}} \cdot \prod_{k=1}^{K} \theta_{\omega_{q}^{k}}^{j^{k}}=\theta_{\hat{\omega}}^{\hat{\jmath}} \cdot \theta_{\hat{\omega}}^{j^{3}} \cdot \theta_{\omega_{q}^{K}}^{\hat{\jmath}} \cdot \prod_{k=1}^{K} \theta_{\omega_{p}^{k}}^{j^{k}}
$$

The validity of (2) for the cycle $\left\{\left(j^{k}, \omega_{p}^{k}, \omega_{q}^{k}\right)\right\}_{k=1}^{K}$ follows immediately. The induction is thereby complete.

If we think of the state as a vector $\omega=\left(t_{1}, \ldots, t_{J}\right)$ of "types" $t_{j}$ of the different players, the assumption that any element of any player's partition intersects any element of any other player's partition is equivalent to the assumption that the state space is equal to the product $\prod_{j=1}^{J} T^{j}$ of type spaces for the different players, so that, for any $j$ and $k \neq j$ and any types $t_{j} \in T^{j}$ and $t_{k} \in T^{k}$, upon observing that his own type is $t_{j}$, player $j$ cannot completely rule out the possibility that player $k$ 's type is $t_{k}$. Types may be correlated, but not to such an extent that the observation of a player's own type allows him to reduce the set of types that are considered to be possible for other agents. For any model exhibiting such a product structure, Proposition 2 asserts that th egiven posteriors are compatible with a common prior if and only if the cycle equations hold for any cycles of length 4 or less.

\section{References}

[1] Yossi Feinberg, Characterizing common priors in the form of posteriors, J. Econ. Theory 91 (2000) 127-179.

\footnotetext{
${ }^{3}$ In Figure 1, this operation replaces the six-edge cycle $1 \rightarrow 3 \rightarrow 6 \rightarrow 5 \rightarrow 8 \rightarrow 7 \rightarrow 1$ by the nine-edge cycle $1 \rightarrow 3 \rightarrow 6 \rightarrow 4 \rightarrow 1 \rightarrow 4 \rightarrow 5 \rightarrow 8 \rightarrow 7 \rightarrow 1$, which consists of the four-edge cycle $1 \rightarrow 3 \rightarrow 6 \rightarrow 4 \rightarrow 1$ and the five-edge cycle $1 \rightarrow 4 \rightarrow 5 \rightarrow 8 \rightarrow 7 \rightarrow 1$. The same operation replaces the five-edge cycle $1 \rightarrow 4 \rightarrow 5 \rightarrow 8 \rightarrow 7 \rightarrow 1$ by the two four-edge cycles $1 \rightarrow 4 \rightarrow 5 \rightarrow 2 \rightarrow 1$ and $1 \rightarrow 2 \rightarrow 8 \rightarrow 7 \rightarrow 1$.
} 
[2] Ziv Hellman and Dov Samet, How common are common priors?, Discussion Paper \# 532, Center for the Study of Rationality, Hebrew University, Jerusalem, February 2010.

[3] Stephen Morris, Trade with heterogeneous prior beliefs and asymmetric information, Econometrica 62 (1994) 1326-1347.

[4] José Alvaro Rodrigues-Neto, From posteriors to priors via cycles, J. Econ. Theory 144 (2009), 876-883

[5] Dov Samet, Iterative expectations and common priors, Games Econ. Behav. 24 (1998),131-141.

[6] Dov Samet, Common priors and the separation of convex sets, Games Econ. Behav. 24 (1998), 172-174. 